\section{Vision für eine Versorgung im Netzwerk: Der Hausarzt in der Suchtmedizin}

Jörg Gölz

Praxiszentrum Kaiserdamm Berlin
Zusammenfassung: Die Vielfalt medizinischer, sozialer und gesellschaftlicher Versorgungsprobleme im Zusammenhang mit der Abhängigkeit von illegalen Drogen kann mit neuen organisatorischen und finanziellen Strukturen besser gelöst werden. Die Vielzahl der Kostenträger, die unterschiedlichen Kostenregelungen in den einzelnen Sektoren und die umstrittenen Zuständigkeiten entfalten eine zerstörerische Dynamik für das koordinierte Zusammenwirken der medizinischen und sozialen Institutionen. Die kammeralistische Finanzierung führt zu Konkurrenz, Abgrenzungs- und Selbstbehauptungskämpfen der so finanzierten Institutionen. Die Folge ist eine Diskontinuität und Desintegration im Versorgungsprozess für den einzelnen Patienten. Zukünftige Versorgungsmodelle und die dazu notwendigen Voraussetzungen werden erörtert.

Schlüsselwörter: Ambulante Suchttherapie - Heroinabhängigkeit - Versorgungsmodelle

A Vision of Integrated Medical Care: The Part of General Practitioners in Addiction Medicine: There is a range of medical and social problems in the care for illicit drug users which can be resolved by new organisational and financial structures. The multitude of financiers, varying provisions for cost refunding in the different sectors and disputes about competences and responsibilities cause a dynamic detrimental to the co-ordinated co-operation of medical and social institutions. Divided financing leads to rivalry between the supported institutions and leaves them struggling for selfassertion and demarcation.

The consequences are discontinuity and disintegration in the process of care for the individual patients. Future models of care systems and their necessary prerequisites are discussed in this article.

Key words: Ambulant Addiction Therapy - Heroin Addiction - Care Systems

\section{Grundsätzliche Probleme der ambulanten Suchttherapie}

Die Möglichkeiten der Diagnostik, Therapie und interdisziplinären Kooperation für Suchtpatienten werden in der ärztlichen Praxis nicht ausgeschöpft. Dafür sind eine Reihe von gesellschaftlichen, psychodynamischen und strukturellen Faktoren verantwortlich:

Gesellschaftlich bedingte Probleme

- Vielzahl der Kostenträger

- Rivalität statt Kooperation

- unsachliche Informationspolitik über Drogen

- politische Vorgaben

Gesellschaftliche Bewertung von Suchtmedizin

- Ausgrenzung der Ärzte („dirty medicine“)

- abwertende Vorurteile („nothing works“)

- unzureichende finanzielle Honorierung

Mängel in Ausbildung und Forschung

- Fehlen einer qualifizierenden Ausbildung

- Fehlen von Behandlungsstandards (evidence based treatment)

- Fehlen realistischer Handlungsanweisungen (good clinical practice)

- Fehlen von Begleitforschung

\section{Gesellschaftlich bedingte Probleme}

Der Prozess der medizinischen und sozialen Rehabilitation wird von mindestens 15 verschiedenen Kostenträgerarten finanziert. Dies führt im Einzelfall zu zeitaufwändigen Prüfungen der Zuständigkeit und der Anspruchsberechtigung.

Kostenträger in der Suchttherapie:

- Gesundheitshilfe der Justiz

- RVO-Kassen, VdAK-Kassen

- Sozialamt

- BfA, LVA

- Seekasse, Bundesbahn-Versicherungsanstalt

- Landesämter für Ausbildungsförderung, Arbeitsamt

- Hauptfürsorgestellen, Versorgungsämter

- Wohnungsämter (landesrechtliche Behörden für Wohngeld)

- Jugendamt, Landesjugendämter

Die Disziplinen, die in der Suchttherapie zusammenarbeiten sollten, stehen häufig in Konkurrenz zueinander. Typische interdisziplinäre Störungen sind die unterschiedlichen Definitionen von Abhängigkeit, der Therapieziele sowie eine unterschiedliche Bewertung der Zielhierarchie. 
Als ein besonderes Problem werden Kompetenzgrenzen, fehlender Respekt für die Fertigkeiten der Einzeldisziplinen und letztlich auch der Kampf um finanzielle Mittel angesehen.

Ein drittes gesellschaftlich bedingtes Problem in der Suchttherapie ist die durch politische Vorgaben bestimmte Informationspolitik über Drogen und die politischen Vorgaben für den Umgang und die Therapieziele bei Abhängigkeit. Kein anderer medizinischer Bereich wird in diesem Umfang von fachfremden Maximen und Regeln beeinflusst.

\section{Gesellschaftliche Bewertung von Suchtmedizin}

Die Dämonisierung des Süchtigen in der Gesellschaft erstreckt sich auch auf deren Behandler [1,2]. Sie werden kurzerhand dem Milieu ihrer Patienten zugeordnet. Brennpunktartig wird dieser Sachverhalt beleuchtet durch den pejorativen Terminus, der sich zum Beispiel in den USA für die Behandlung Drogenabhängiger eingebürgert hat: „dirty medicine“.

Unter den niedergelassenen Ärzten sind vor allem die hausärztlich tätigen Allgemeinmediziner und Internisten die erste Anlaufstelle für süchtige Patienten. Gerade sie haben häufig eine mangelhafte suchtmedizinische Ausbildung. Die Kontakte mit Süchtigen in der klinischen Ausbildung verstärken eher die Neigung, im späteren Berufsleben die Behandlung Suchtkranker zu vermeiden. Der daraus resultierende Mangel an Fertigkeiten im angemessenen Umgang mit Abhängigen führt dazu, dass die typischen Interaktionsstörungen zwischen Suchtpatienten und Arzt nicht aufgelöst werden können. Dies mündet in eine Spirale negativer Lernerfahrungen: „nothing works“.

Die Honorierung medizinischer Leistungen besitzt eine selektive Wirkung auf ärztliche Weiterbildung und die Neigung, problematische Patientengruppen zu behandeln. Präventive, motivierende, diagnostische und therapeutische Maßnahmen bei Suchtkranken sind sehr zeitaufwändig. Da ärztliche Arbeitszeit im Vergleich zu technischen Leistungen schlecht honoriert wird, bedeutet sachgerechte Suchtbehandlung auch immer ein finanzielles Risiko. Folge sind generelle Ablehnung solcher Patienten und eine minimale diagnostische und therapeutische Aktivität.

\section{Mängel in Ausbildung und Forschung}

Die geringe Wertschätzung der ambulanten Suchttherapie durch niedergelassene Ärzte dokumentiert sich in erheblichen Mängeln bei der Ausbildung, der Erarbeitung von Behandlungsstandards und standardisierten Handlungsanweisungen in der Praxis sowie deren fortgesetzter Veränderung durch Forschung.

Nur durch eine berufsbegleitende Qualifikation auf dem Gebiet Suchtmedizin wird ein professioneller Umgang mit den Patienten erlernt [3] und gleichfalls dokumentiert, dass es sich bei der Behandlung Süchtiger um ein definiertes Feld ärztlicher Tätigkeit handelt.
Das Fehlen von diagnostischen und therapeutischen Standards und Handlungsanleitungen im Sinne von „evidence based treatment“ und „good clinical practice“ [4] führen zu einer Beliebigkeit der praktischen Arbeit. Dasselbe gilt für die Dokumentation [5].

Wegen dieser Unübersichtlichkeit meidet die suchtmedizinische Forschung den ambulanten Bereich der Suchttherapie. Der Mangel an Standardisierung lässt kaum verwertbare Ergebnisse erwarten. Gleichzeitig besteht der Vorwurf von Seiten der Forschung, die niedergelassenen Ärzte seien nicht in der Lage, wichtige Erkenntnisse der Suchtforschung in praktisches Handeln umzusetzen [6].

\section{Notwendigkeit, Voraussetzungen und Widerstände gegen interdisziplinäre Kooperation}

Die Organisation des Versorgungssystems mit Fokussierung auf Teilaspekte einer biografischen Entwicklung zerstört den Zusammenhang aller Teile in einem unteilbaren Individuum. Die einzelne Versorgungsinstitution verabsolutiert den von ihr bearbeiteten Aspekt und vernachlässigt damit zwangsläufig die wechselseitigen Einflüsse der einzelnen gestörten Funktionen und Lebensbereiche. Was sich in der Lebenswirklichkeit als ein dynamischer Prozess verschiedener Störungen darstellt, wird im aufgeteilten Versorgungssystem zwangsläufig als unverbundenes Nebeneinander isolierter Schädigungen bearbeitet. Therapieprozess und therapiertes Individuum bleiben sich fremd, da sie nicht die gleiche Grammatik benutzen.

Nur eine interdisziplinäre Kooperation kann diesen Mangel des Versorgungssystems ausgleichen.

Notwendigkeit zur Kooperation der Disziplinen

- Schädigungssyndrome überschreiten Fachgrenzen

- verschiedene Kostenträger mit Abgrenzung der Zuständigkeit

- gemeinsame Auseinandersetzung mit dem Thema

- dynamischer Wissensaustausch

- Beeinflussung der Standpunkte

- Optimierung des Behandlungsprozesses

- beschleunigter Entscheidungs- und Planungsprozess

Drogenabhängigkeit geht mit einer Vielzahl von Schädigungssyndromen einher (toxikomanes, psychisches, somatisches und soziales Syndrom), für deren Behandlung verschiedene Disziplinen eng kooperieren müssen. Nur die gemeinsame Entwicklung von Handlungskonzepten, der Austausch von spezialisiertem Wissen und die Modifizierung der Einzelperspektiven können einen stringenten Behandlungsprozess hervorbringen.

Die Angehörigen der einzelnen Disziplinen haben weit auseinander liegende Qualifizierungen, Berufserfahrungen und Blickwinkel auf das Kooperationsziel. Allein die Aufzählung der Berufe macht dies deutlich (Tab. 1). 
Tab. 1

\begin{tabular}{ll}
\hline Nichtmedizinisch & Medizinisch \\
\hline Sozialarbeiter/-pädagogen & Suchtmediziner \\
Mitarbeiter Ämter & Psychiater/Familientherapeuten \\
Krankenschwestern & Infektiologen (HCV, HIV, HBV) \\
Sprechstundenhilfen & Gynäkologen \\
Bewährungshelfer & Geburtshelfer \\
Angestellte Sozialhilfe & Kinderärzte \\
Rehafachkräfte & Laborärzte \\
häusliche Krankenpflege & Transplantationsmediziner \\
Psychologen & Ärzte Haftkrankenhaus \\
Mitarbeiter der Selbsthilfegruppen &
\end{tabular}

\section{Kooperierende Disziplinen}

Schon zwischen den Vertretern der gleichen Berufsgruppe bestehen erheblich unterschiedliche Auffassungen über Prioritäten. Noch prononcierter ist dies der Fall zwischen den Vertretern der unterschiedlichen Disziplinen. Dadurch ergeben sich häufig schon vor jeder Kooperation Widerstände gegen ein Kooperieren, soweit es nicht unumgänglich ist.

\section{Widerstände gegen interdisziplinäres Arbeiten}

- Angst vor Verlust der Autonomie (Entscheidungen)

- Angst vor Verlust der Identität (neue Identität)

- Angst vor Hierarchien (Leitliniendebatten)

- Angst vor Konkurrenz (Leistungsvergleiche)

- Angst vor Verantwortung (Finanzierung)

- Angst vor dem Prozess der Kompetenzabgrenzung

- Aversion der Kostenträger gegen Mischfinanzierungen
Diese Widerstände bleiben ohne Bearbeitung und institutionelle Organisation während des Kooperationsprozesses ständig erhalten und wirken sich lähmend auf den Behandlungsprozess aus. Die Aufspaltung der Finanzierung der einzelnen Behandlungsschritte auf verschiedene Kostenträger verstärkt noch die desintegrierende Wirkung der psychologischen Widerstände.

Das bedeutet, dass sich aus dem Nebeneinander verschiedener Disziplinen nicht ohne weiteres eine kooperierende Gemeinschaft entwickelt. Die Lebenswirklichkeit der Drogentherapie in den großstädtischen Ballungszentren spiegelt diese Lage wider: ein schlecht verbundenes System von Einzelgruppen mit marginaler Kooperation überall dort, wo man selbst ohne die Hilfe einer anderen Gruppierung nicht mehr vorwärts kommt.

Der notwendige Integrationsprozess bedarf eines großen logistischen Aufwands: sowohl zur Herstellung der Kooperation als auch zur Aufrechterhaltung der Zusammenarbeit.

Neben einer Einigung über den Inhalt der Therapieziele gehört dazu eine Bestimmung der finanziellen Möglichkeiten, der Aufbau einer organisatorischen Struktur mit Verantwortlichkeiten und fachlicher Hierarchie durch Institutionalisierung der Kooperationsformen und durch vermittelnde Gremien, die Aufrechterhaltung des zielgerichteten Funktionszustandes der kooperierenden Einzeldisziplinen durch Teamentwicklung und vertrauensbildende Kommunikation. Gleichzeitig muss dieser funktionsfähige Binnenzustand ständig neuen wissenschaftlichen, gesellschaftlichen und politischen Entwicklungen angepasst werden.

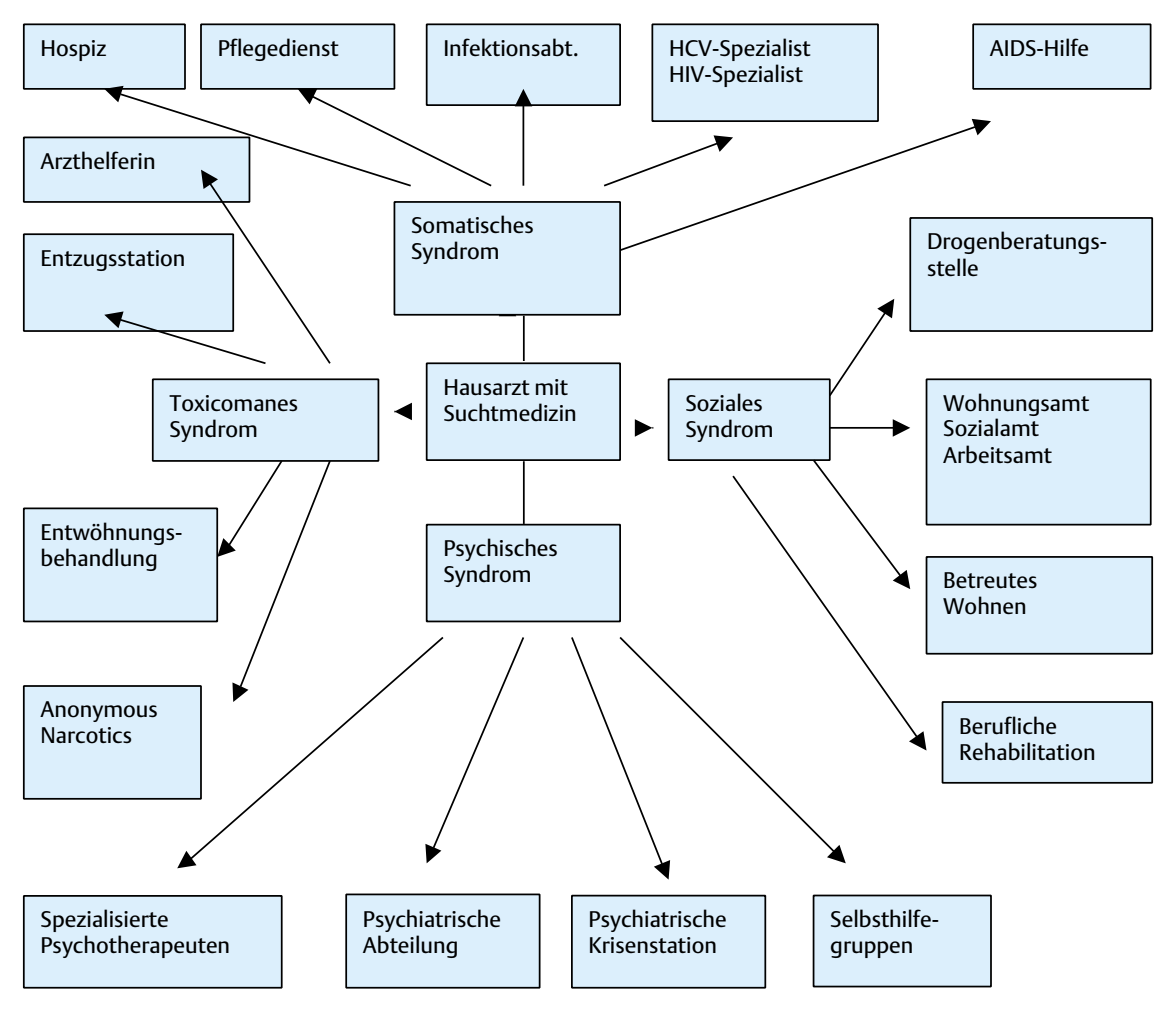

Abb. 1 Versorgungsnetz für den suchtmedizinisch tätigen Hausarzt. 
Tab. 2

\begin{tabular}{lll}
\hline Drogenberatung & gemeinsamer Bereich & Medizin \\
\hline - Streetwork & - Therapieplanung & - Notfalltherapie \\
- niedrigschwellige Einrichtungen & - Entwöhnungsbehandlung ambulant/stationär & - Basisdiagnostik \\
- harm reduction & - berufliche Rehabilitation amb., teilstat., stat. & - Substitution \\
- Drogenberatung & & - somatische Therapie \\
- PS der Substitution & & - psychiatrische Therapie \\
- Nachsorge & & - Entzugsbehandlung \\
- Betreutes Wohnen & - Psychotherapie
\end{tabular}

\section{Voraussetzungen zur Kooperation}

- Definition des Therapiezieles

- Analyse der Ressourcen

- Organisationsstruktur

- Organisationsprozess (Gruppenverhalten, Vertrauen, Kommunikation)

- Führung

- Teamentwicklung

- Dynamik von Handlungsgrundsätzen

- Institutionalisierung der Kooperationsformen und Gremien

\section{Modelle einer integrierten Versorgung Hausärztliche Vernetzung}

Jenseits von großstädtischen Ballungsräumen ist der suchtmedizinisch tätige Hausarzt die Steuerungsfigur für die Koordination der einzelnen Aufgaben während der Suchtbehandlung. Er muss Kontakt zu einem breit aufgefächerten Versorgungsnetz halten.

\section{Spezielle Versorgungsmodelle}

Zentral sind zwei Berufsgruppen an der Versorgung Opiatabhängiger beteiligt: Ärzte und Drogenberater. Beide Gruppen haben klar unterschiedene alleinige Kompetenzen und ein Arbeitsfeld mit gemeinsamer Kompetenz (Tab. 2).

\section{Aufgabenverteilung}

Die Kooperation dieser beiden Berufsgruppen bildet den Kern für spezialisierte Versorgungsnetze, die sich überwiegend nur in großstädtischen Ballungsräumen verwirklichen lassen.

Für großstädtische Ballungsräume wird in Zukunft auch der Zusammenschluss mehrerer bisher selbständiger Versorgungssysteme zu einer organisatorischen Einheit möglich sein. Dann werden vom niedrigschwelligen szenenahen Kontaktladen bis zur stationären beruflichen Rehabilitation sämtliche Versorgungsstufen innerhalb eines Verbundes möglich sein.

Das soll am Beispiel des in Berlin geplanten Versorgungsmodells dargestellt werden.

\section{Teilnehmer am Berliner Modell der integrierten Versorgung}

- 6 suchtmedizinische Schwerpunktpraxen

- 2 stationäre Abteilungen

- 1 gynäkologisch/geburtshilfliche Abteilung
- 6 niedrigschwellige Kontakteinrichtungen

- 3 szenestationierte med. Versorgungsmobile

- 11 Drogenberatungsstellen

- 7 Abteilungen für ambulante und stationäre Abstinenztherapie

- 6 Abteilungen für ambulante und stationäre Rehabilitation

- 11 Abteilungen für Wohnen und Nachsorge

\section{Leistungen des Berliner Modells}

1. Harm reduction

- Verhaltensänderung der Konsumgewohnheiten

- niedrigschwellig Kontakt

- niedrig Unterstützung

- niedrigschwellige Diagnostik und Prävention

- Drogennotfalltraining

2. Medizinische Intervention

- Substitution mit PSB

- qualifizierter Entzug

- ambulante und stationäre Entwöhnung

- Rückfallprophylaxe

- Therapie chronischer Infektionskrankheiten

- Therapie psychiatrischer Komorbidität

3. Soziale und berufliche Rehabilitation

- finanzielle Basisversorgung

- Rechtsberatung, Schuldenregulierung

- Wohnen (einzeln, betreut, in Nachsorge)

- Schulabschluss

- Zusatzqualifizierung

- Berufsausbildung

$\mathrm{Zu}$ den Zielen der Kooperation gehören eine patienten-/ klientenorientierte Organisation und eine qualifizierte Versorgung von Subgruppen (Migranten, Schwangere, Komorbidität) ebenso wie richtlinienorientierte Interventionen aller Disziplinen, eine standardisierte Datenerhebung und die Ausweitung der Versorgungsforschung.

Diese Ziele umzusetzen erfordert eine abgestimmte Organisation. Die Organisationsmerkmale der Kooperation sind daher die Bildung einer gemeinsamen Rechtsform, der Aufbau einer gemeinsamen Verwaltung, eine gemeinsame Finanzplanung sowie ein modellgebundenes Konsortium der Kostenträger. 


\section{ambulant}

\section{HIV-Schwerpunktpraxen}

HCV-Schwerpunktpraxis

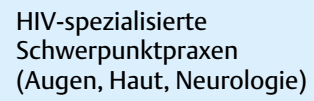

HIV-spezialisierte Schwerpunktpraxen (Augen, Haut, Neurologie)

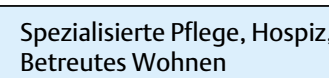
Betreutes Wohnen

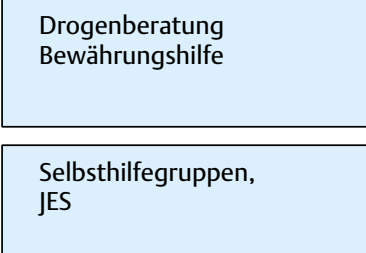

teilstationär
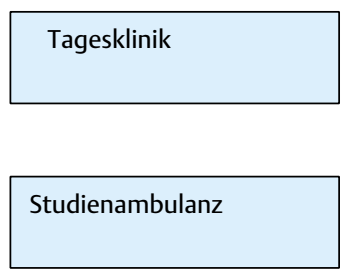

\section{Hepatologische Ambulanz}

stationär

Infektionsabteilung

Chirurgie

Abteilung für stationäre Entzüge

Geb.-gynäkologische Station

Krankenhaus Haftanstalten

Transplantationszentrum
Abb. 2 Versorgungsnetz HIV-/HCVinfizierte IVDU.

\section{Ausblick}

Ein organisatorisch und finanziell eigenständiger Verbund aus präventiven, medizinischen und rehabilitativen Versorgungsinstitutionen kann die Betreuung von Drogenkonsumenten kompetenter, differenzierter, wirkungsvoller und kostengünstiger lösen. Die Umstrukturierung eines kostenträger- und institutionsorientierten Versorgungssystems zu einer patientenorientierten Organisation hat erhebliche Widerstände $\mathrm{zu}$ überwinden: Angst vor Verlust von Entscheidungsautonomie und Eigenständigkeit, Angst vor der Übernahme einer finanziellen Verantwortung und die Aversion der Kostenträger gegen mischfinanzierte Versorgungsprojekte.

Der wesentliche Vorteil eines solchen Verbundes liegt darin, alle Therapieansätze unter einem Dach zu vereinen und damit für den Patienten jeweils rasch die Maßnahme zur Verfügung stellen zu können, die seiner aktuellen Problemlage entspricht. Da Abhängigkeitserkrankungen überwiegend mit immer wiederkehrenden Rückfällen verlaufen, wechseln auch die Problemlagen rasch. Für die soziale Prognose des Abhängigen ist der jeweils rasche Kontakt zum Hilfesystem von überragender Bedeutung.

\section{Literatur}

${ }^{1}$ Macqueen AR. Why general practitioners might avoid drug and alcohol work. Drug Alcohol Rev 1997; 16: 429-431

${ }^{2}$ Newman RG. What's so special about methadone maintenance? Drug Alcohol Rev 1991; 10: 225-232

${ }^{3}$ Roche AM, Guray C, Saunders JB. General practitioners experiences of patients with drug and alcohol problems. Br J Addiction 1991; 86: 263-275

${ }^{4}$ Hall W. Evidence based treatment for drug misuse: brigding the gap between aspiration and achievement. Addiction 1997; 92: 373-374

${ }^{5}$ Lehnitzk C, Simon R. Systembeschreibung SEDOS München: IFT Institut für Therapieforschung, 1994; Version 1.2 (IFT Manuale Bd. 27)

${ }^{6}$ Hall W. Making research more relevant to practice. Drug Alcohol Rev 1995; 14: 149-150

Dr. med. Jörg Gölz

Praxiszentrum Kaiserdamm

Kaiserdamm 24

14057 Berlin 